\title{
Quelle place dans quelle famille: que disent les parents de leurs enfants?
}

Patricia Bessaoud-Alonso ${ }^{\star}$

Université de Limoges, Limoges, France

\section{Résumé}

La norme produit-elle un « mauvais » ou un «bon » enfant inscrit dans des configurations familiales et sociétales en mutation? Produirait-elle des enfants à leur place dans la relation qu'ils entretiennent à la génération précédente? Les pratiques éducatives familiales, au croisement des injonctions sociétales - conscientes et inconscientes - et des petits arrangements familiaux entre conflits et sérénité, déclenchent des mouvements constants qui laissent, parfois, les parents et les enfants déstabilisés par un "jeu » d'interférences entre égalité et hiérarchie, ancré à un maillage institutionnel.

Mots clés: parents; enfants; norme; institution; socio-clinique.

\section{Which role in which family? What do parents say about their children?}

\begin{abstract}
Does the norm create a "bad" or a "good" child in the context of a constantly changing family and society? Does it, perhaps, create children as they should be as regards their relation with the previous generation? Educational practices within the families take place where societal injunctions - either conscious or unconscious - and compromise solutions between conflict and serenity meet. These practices lead to constant movements that sometimes make parents and children feel confused in a "sort of game" of interferences between equality and hierarchy, tightly tied to an institutional networking.
\end{abstract}

keywords: parents; children; norm; institution; clinical sociology.

\section{Deux dispositifs de recherche}

\section{Des ateliers de circulation de la parole}

Pendant près d'un an, deux groupes se sont réunis en « ateliers ${ }^{1}$ dans le cadre de la Recherche-Action « en associant leurs parents, tous les enfants peuvent réussir» que la municipalité qui nous a sollicités a soutenu en réunissant les organismes publics, les associations et les établissements scolaires qui souhaitaient participer à une meilleure compréhension des problèmes que rencontrent les parents et les professionnels qui contribuent à l'éducation des enfants sur ce territoire.

Il s'agissait, dans un premier temps, de permettre, d'une part, un échange entre parents, et, d'autre part, entre professionnels afin que chacun puisse signifier en toute confiance et en toute liberté comment il vivait son expérience éducative.

Les séances, animées par trois personnes, étaient enregistrées puis retranscrites ${ }^{2}$ afin que chacun des participants puisse reprendre ses propos pour les corriger ou les enrichir. Si les parents se sont assez volontiers prêtés à ce jeu, il n'en a pas été de même avec les professionnels qui ont souvent déserté le champ du vécu pour proposer des débats plus généraux. L'analyse de ces résistances a largement contribué à faire avancer la compréhension des problèmes et leur dévoilement.

\footnotetext{
^Adresse postale : Université de Limoges - Faculté des Lettres et Sciences Humaines, 39 E, rue Camille Guerin. 87000 - Limoges.E-mail : patriciaalonso1@gmail.com ${ }^{1}$ Cadrage des ateliers : Un groupe de parents et un autre de professionnels de l'éducation. L'animation était assurée par deux universitaires, une psychologue et une coordinatrice de la MDA. Chaque atelier était co-animé par trois personnes. Mise au point d'un contrat garantissant les conditions de confidentialité des échanges. ${ }^{2}$ Toutes les retranscriptions ont été anonymées.
}

Après cinq rencontres chaque groupe a établi une liste de points que ses membres souhaitaient mettre en discussion. Ainsi a-t-on abouti à une première « production de savoirs » qu'il est devenu possible de « croiser », les parents exprimant leurs constats aux professionnels, les professionnels adressant aux parents leurs propres préoccupations. Puis, deux réunions ont été consacrées à cet " échange de savoirs » et on aurait pu en programmer d'autres, tant le débat s'est révélé fécond.

La pratique de l'écriture a été encouragée par les animateurs de l'atelier, modestement, puis avec davantage d'ambition pour quelques participants. Cette pratique a été favorisée par la sollicitation des animateurs, en fin de séances, en amenant chaque groupe à produire une liste de neuf thèmes à débattre. Cette démarche a été renforcée en communiquant à chacun les retranscriptions des interventions qu'il avait faites et en lui proposant de les retravailler. La séance d'accompagnement individualisé à l'écriture a permis, entre autres, aux mères d'origine étrangère, d'expliciter leur propos, un animateur assurant le secrétariat à cette occasion.

\section{Des temps formels et informels pour dire}

Dans le cadre du dispositif de soutien à la parentalité, lancé par la $\mathrm{CAF}^{3}$ la commande a été de construire un diagnostic partagé sur le territoire de la Haute-Vienne afin de renforcer la cohérence et la coordination des réponses aux attentes des familles dans le champ du soutien à la fonction parentale. L'enjeu prioritaire a été

\footnotetext{
${ }^{3}$ Caisse d'allocations familiales (organisme privé chargé d'une mission de service public qui concerne la famille : soutien financier, technique et d'accompagnement, logement et vie sociale, solidarité, insertion sociale et professionnelle, familles confrontées à la maladie et au handicap...)
} 
de cerner ce qui favorise la participation des parents aux actions et de susciter les initiatives familiales. La dimension retenue est préventive et repose sur l'étude du lien entre un parent et un enfant, quelle que soit la structure familiale dans laquelle il évolue, dans le but d'assurer le soin, le développement affectif, psychologique, et l'éducation de l'enfant.

Notre méthodologie de recherche s'inscrit dans une démarche socio-clinique. C'est-à-dire une approche compréhensive des situations vécues par les acteurs/ sujets - parents et professionnels -, en articulant la dimension sociale dans des contextes institutionnels et socio-historiques précis et les vécus, les faits subjectifs, qui construisent la réalité, la vision du monde des sujets. « Tant il est vrai que plus on va vers le subjectif, plus on trouve et retrouve le social » (REVAULT D'ALLONNES, 1989, p. 30).

Une approche d'inspiration ethnographique, consistant en une observation fine et systématique « de tout espace, de tout objet, de tout comportement (geste et parole) qu'ils soient repérés en dehors d'une situation d'entretien ou à son occasion » (WEBER, 2009) a également permis de recueillir un certain nombre de données lors des entretiens ou de rencontres plus informelles. Elle a permis, au delà des mots, d'affiner et de nourrir nos interprétations et notre travail d'analyse.

Sept entretiens ont été réalisés avec des parents de 20 à 50 ans, dont les enfants ont entre un mois et dix-sept ans. Là encore plusieurs catégories socioprofessionnelles sont représentées :

- deux familles monoparentales de classes moyenne et inférieure,

- une mère sans emploi ayant un fils handicapé,

- deux mères de classe moyenne et une de classe moyenne supérieure,

- une famille de classe moyenne supérieure avec un enfant handicapé.

Une lecture des documents institutionnels et des données sociodémographiques a permis d'identifier les spécificités et problématiques propres à chaque territoire et de compléter les données collectées et/ou mises à disposition par le comité départemental.

Le « faire famille » (CADORET, 2002) a renvoyé à une interprétation multiple liée à la réalité concrète des situations et des figures symboliques et réelles du père et de la mère. Mais ce « faire famille » n'a été que très peu énoncé en tant que tel. La place, en particulier dans le dispositif des ateliers, a été en grande partie une place du « je » de la mère. Quand la famille a été convoquée elle l'a été par le prisme d'une entité singulière, particulière, comme dissociée de tout processus institutionnel.

L'émergence du père est apparue du coté des familles monoparentales étrangères, alors qu'il est territorialement absent. Cette figure paternelle a été présentée comme père et mari, occupant une place au sein de la famille, le père absent devenant le père présent dans le dis- cours de ces femmes. Les autres femmes ont passé sous silence le père, le conjoint, comme figure non présentée, non représentée, comme si le « je » de la mère devenait un « je » de la famille où les pères ne semblaient pas apparaître dans un « nous ».

La pratique de la parentalité (SELLENET, 2012) s'est exprimée du coté des mères car dire c'est faire (AUSTIN, 1991[1962]). Le « nous » de la famille, quand il a été explicite, est un « nous » fondé sur la relation à l'enfant. La parole qui a circulé , à travers le récit d'expérience quotidienne, a pointé la difficulté d'être parent du point de vue des parents eux-mêmes. Celle-ci s'est énoncée dans tous les cas, parents engagés ou non dans les institutions éducatives, sous le registre de la suspicion, de la peur, de la protection. Crainte de confier son enfant : « aura -il la même éducation que celle dispensée dans la famille?", suspicion : "je vais être jugée comme parent? Je ne fais pas bien, pas comme il faut? ». Est-ce le reflet d'une perte de confiance du statut d'adulte ? Où une redéfinition de ce statut en perpétuel mouvement?

L'expérience subjective de la parentalité a mis en exergue un certain nombre de questionnements sur la relation des parents vis-à-vis des institutions et de leurs enfants. Comment le parent évoque-t-il son enfant ? Est-il l'enfant rêvé, réparateur, persécuteur ? L'enfant fait-il famille (DIASIO, 2009) ?

L'idée d'être un «bon parent » peut-elle s'entendre à travers les injonctions, les pressions faites aux familles, ainsi qu'à la place que l'enfant du désir (GAUCHET, 2004) négocie, s'octroie, construit dans l'espace familial ?

Nous avons travaillé sur plusieurs pistes d'interprétation et d'analyse de la parole des parents dans le cadre de ces recherches. Je m'intéresserai ici à la manière dont les parents racontent leurs enfants, expriment leurs visions éducatives et du monde, les paradoxes et controverses qui les traversent et les effets qu'ils produisent : effets intra-familiaux, intersubjectifs, imaginaire collectif (CASTORIADIS, 1999[1975]).

\section{C'est mon enfant ?}

Pour les parents, c'est par un certain nombre de termes que la mise en scène de la relation à leurs enfants s'est construite. Le premier s'est bâti autour de la protection : protéger les principes éducatifs de la famille, protéger d'éventuelles malveillances. Le second rend compte des inquiétudes dues à la séparation et à une certaine difficulté de passer le relais à un tiers. Enfin, la méfiance concentrée sur les institutions éducatives et l'empêchement qu'elles engendrent pour le parent de maîtriser l'ensemble des espaces dans lesquels évolue l'enfant. Ce qui, pour certaines mères, a signifié une forme de perte. Perte ou contrôle ? La question est posée ! Cela pose également la question formulée par Martine Segalen (2010): à qui appartiennent les enfants?

Nous avons également pu noter la fréquence du terme de « bricolage » comme la métaphore d'un ordre social bricolé dans la relation parent-enfant et dans leurs interactions. Cependant, ce « bricolage » n'est-il pas un perpétuel mouvement entre créativité et normativité ? 
Agnès est une jeune femme dynamique, très engagée dans le tissu associatif local. Elle est la mère d'une petite fille de 4 ans, que je nommerai Lou, scolarisée depuis un an. Elle décrit des faits de la vie quotidienne où elle met en scène sa fille et la relation qu'elle entretient avec elle. Lors d'un atelier, elle raconte qu'elle est persuadée qu'une rentrée scolaire en janvier pour sa fille est la meilleure solution et qu'elle (la mère) serait prête. Le moment venu, elle pense que c'est impossible qu'une seule personne, l'enseignante, puisque s'occuper seule de tous les enfants de la classe. Elle exprime son angoisse face à l'abandon présumé. Aucun adulte pour la protéger, la rassurer. Elle ne pourrait plus rien contrôler et une partie de la vie, de son enfant, allait totalement lui échapper. Le jour J Lou entre dans la classe d'un pas décidé. De retour à la maison, elle explique à sa mère que la cuisine dans la classe est plus grande que celle de sa chambre. La mère hésite les jours suivants à acheter des objets, des jouets identiques à ceux de l'école.

Ce que soulève Agnès est avant tout la difficulté de la séparation lors du passage de l'enfant vers un autre espace que celui du territoire familial. L'espace scolaire est présenté comme dangereux, inquiétant, incontrôlable. Elle propose une forme de continuité des espaces à travers la cuisine de la classe et celle de la maison afin de recréer un univers familier pour l'enfant. Sauf que l'école maternelle joue un rôle essentiel. Elle fait rupture avec la communauté familiale comme un moyen de se développer, de découvrir de nouveaux mondes, pour revenir progressivement plus libre dans l'espace familial (MEIRIEU, 2008). En ce sens la cuisine devient un objet transitionnel (WINNICOTT, 2002[1971]). Au delà, de ces aspects psycho-affectifs, se pose la question de l'institution. L'institution est-elle signifiante ? Contenante ? Fait-elle sens comme une structure collective sur laquelle l'individu s'appuie, se tient? Le paradoxe que l'on peut souligner est la dichotomie entre un processus d'individualisation du rapport au monde et l'ambivalence de l'attachement au sens de Bowlby (2002) transposé aux institutions. Ce que décrit et analyse Bowlby (2002) dans sa théorie de l'attachement, serait ici représenté par l'institution (LOURAU, 1970) comme socle fondamental de l'organisation des relations humaines.

\section{Être autonome? Avoir des droits ?}

La notion d'autonomie est associée au libre abrite, à la construction de soi et à la formation personnelle. De la maison à l'école, du centre de loisirs aux médias, les parents, les professionnels, les discours institutionnels évoquent l'importance de l'autonomie des enfants. Être autonome à la crèche, être autonome à la maison, être autonome à l'école... La liste est longue ! Dans tous les espaces institutionnels où évolue l'enfant c'est un leitmotiv. L'autonomie est devenue la voie à suivre au risque de s'y perdre ! Alors qu'en droit l'enfant est dans l'incapacité d'être autonome, la définition juridique de la personnalité est la capacité à être titulaire de droits et d'obligations. Pour autant, l'incapacité de l'enfant ne le prive ni de droits ni d'obligations mais ne lui permet pas d'en avoir l'exercice. En ce sens l'enfant n'a aucune autonomie. Toute- fois, la loi ${ }^{4}$ prévoit que dans leur exercice de l'autorité parentale, «Les parents associent l'enfant aux décisions qui le concernent, selon son âge et son degré de maturité. » (FRANCE, 2002, article 2). Comment les parents se saisissent de cette notion plurielle et se positionnent entre autonomie de l'enfant et droits de l'enfant?

Chantal a deux enfants, un fils de onze ans et une fille de cinq ans. Elle relate un événement qui s'est produit autour de l'habillage. La petite fille décide de mettre une robe bleue et légère et refuse ce que sa mère lui propose : un pantalon et un pull. C'est l'hiver et il fait froid. L'enfant n'accepte pas de s'habiller. La mère s'incline en lui expliquant qu'elle ne veut pas entendre une seule fois qu'il fait froid dehors. Elle explique sa décision de la manière suivante : «Je trouve que c'est très bien, ça lui apprend l'autonomie ».

L'intervention de Chantal va produire des réactions vives sur les autres participantes dru groupe. Catherine a été la première à réagir :

Alors, là, je suis dépassée. Sans doute mon statut de grand-mère! Tout de même, et sans jugement de valeur ! C'est quand même pas à une gamine de cinq ans de décider toute seule de la façon de s'habiller. Tu dis : “- Je ne veux pas t'entendre dire j'ai froid", mais c'est à toi d'évaluer ce genre de choses. Je ne vois pas trop où se trouve l'autonomie là-dedans?

Siti a 5 enfants et les éduque seule, son mari est retourné au pays. Elle prend à son tour la parole :

C'est moi qui dit car je suis la maman! Je dois dire des choses comme ça car à la maison tous les jours, je suis "tout", la maman et le papa. Mon fils, il ne sait pas trop : je veux, je ne veux pas, c'est comme ça! Il n'est pas content et me dit "je suis grand et je peux choisir mon sac. Mes copains ils choisissent". Mais moi, je lui dis : t'as pas besoin d'un sac. C'est tout! et puis je ne discute plus avec lui.

La séance s'est poursuivie dans une confrontation parfois vive. Cependant, les participants ont pu exprimer leur point de vue et en débattre. L'intervention de Chantal pose la question de la place de l'enfant - Relation horizontale ? Relation verticale ? - et l'instauration de nouveaux rapports entre parents et enfants. Dans les années 70 Girard (1957) avait observé les débuts d'une forme de démocratie familiale articulée aux changements sociétaux : plus de démocratie, moins d'autoritarisme. Qu'en est-il aujourd'hui ? Les échanges précités mettent en lumière la complexité de l'exercice de la démocratie dans la famille. L'enfant a droit à une éducation qui lui permet d'intérioriser les règles sociales et morales, mais avant tout une éducation qui favorise son épanouissement personnel. Cette double injonction faite aux parents modifie leurs comportements éducatifs. Elle les modifie pour qui ? Pour eux-mêmes ou pour l'enfant ? Les parents sont souvent traversés par le bien fondé de leurs pratiques, « ballottés » entre leurs propres désirs, ceux de leurs enfants et leurs choix de vie. C'est sans doute dans cet interstice que le bat blesse ! De quoi sont porteurs les parents? De culpabilité face au modèle que leur impose

${ }^{4}$ Code civil loi du 4 mars 2002 relative à l'autorité parentale. 
la société : être un bon parent. C'est à dire aimer son enfant fabriqué par amour, ne rien lui refuser au nom de celui-ci, le protéger de tous les maux, de toute souffrance, le maintenir dans un cocon et à la fois l'amener de plus en plus tôt « hors de l'enfance ». Comme pour justifier l'instauration d'une parole enfantine à prendre en compte à égalité de celle du parent. C'est dans ce cadre, au delà de la relation intersubjecitve enfants-parents, mais confrontés aux institutions éducatives, que les parents ont intériorisé, comme un allant de soi, l'autonomie de l'enfant. Cependant, cette intériorisation n'est pas une règle absolue dans les pratiques familiales instituées. Souvent, ce n'est qu'un mot dénué ou détourné de sa signification. L'autonomie est une notion polysémique pour les parents et pour les enfants. Un reflet que renvoie l'enfant au parent sur sa capacité à fabriquer un «bon enfant ».

\section{Une norme éducative ?}

Il existe autant de pratiques que de familles : pratiques liées aux temporalités professionnelles, aux activités de loisirs, aux contraintes domestiques, marquées par des mutations sociétales et des configurations familiales multiples. La famille contemporaine se trouve confrontée à une construction multiforme de leurs enfants dont ils n'ont pas forcément conscience avant l'adolescence (NEYRAND, 2014). Ce qui entoure l'enfant dès le plus jeune âge, en particulier l'environnement médiatique, a des conséquences sur la suprématie des parents comme fondement unique d'une norme éducative ou de principes éducatifs. Cependant, il serait naïf de penser que l'influence du social et des milieux dans lesquels les enfants évoluent - origine des parents, environnement spatial et temporel, forme familiale (les enfants peuvent parfois vivre dans et entre deux cellules familiales distinctes, aux rythmes et fonctionnements différents)... - soit obsolète.

Cette question d'une « norme éducative » s'est focalisée, dans nos recherches, autour du repas et les manières de faire, ensemble ou séparés : repas pris autour de la table, devant un écran de télévision « au plateau », un menu identique pour toute la famille en privilégiant une cuisine variée et saine, à la carte afin d'éviter les conflits, dans une temporalité variable en fonction des activités des uns et des autres. Une diversité des modalités de prise des repas relevant davantage de la structure et de l'organisation familiale que d'un modèle social dominant.

L'exemple de la table est assez significatif d'un modèle de société à un instant donné. Si autrefois les repas étaient une « institution » bien ordonnée où chacun à sa place respectait un ordre social, celle des enfants les reléguait au silence. Aujourd'hui, le repas prend des formes très variées au quotidien. Il satisfait la demande individuelle : « chacun mange ce qu'il souhaite ». Il accorde à l'enfant une place collaborative et un temps éducatif pour la mère. Il permet d'éviter les conflits. Cela signifie t-il moins de normativité ou alors un déploiement plus large de celle-ci ? Il semblerait que le temps du repas reste un moment de rassemblement important pour les individus. Ce temps oscille entre uniformisation et individualisation (ELIAS, 1989). Il se forme dans la relation sujet-société un mou- vement permanent mais également dans les implications subjectives aux institutions. La normativité des comportements et des pratiques n'est pas une illusion du passé et la désinstitutionnalisation de la famille, où plutôt les configurations actuelles de celle-ci, n'est pas davantage fondée. La place et le statut de l'enfant ont changé, c'est indéniable. Il est autorisé à exprimer ce qu'il souhaite, il devient un partenaire pour certains parents. Il bénéficie souvent d'un espace à lui dans la maison. Il a des droits et est une personne à part entière. Ses parents l'ont désiré pour lui-même. L'état le protège, répare les défaillances parentales et dans le même temps soutient et accompagne le parent à travers des dispositifs financiers, techniques, éducatifs et psychologiques d'exercice de la parentalité.

La relation des parents à leurs enfants répond aux normes éducatives actuelles ancrées dans un temps et un espace donné où le « je » de la famille exprime une forme hyper-individualisée et plurielle de faire famille centrée sur l'enfant. La famille contemporaine « bricole » sa pratique parentale dans un monde complexe, économiquement et politiquement imprévisible et où les institutions apparaissent parfois instables et peu structurantes tout en étant omniprésentes.

\section{Conclusion}

Les recherches ont mis en évidence que les parents étaient soucieux de la parole de leurs enfants. Parole à laquelle ils tentent d'apporter des réponses en accord avec leur interprétation d'une relation éducative juste. Ils disent « leur enfant " avec leurs mots, des mots balisés de l'empreinte des modèles et des normes actuelles. La multiplicité des normes éducatives n'est pas que le reflet de la diversité du « faire famille ». Elle nous raconte la vision du monde des individus où l'enfant d'aujourd'hui, comme celui d'hier, est porteur d'un devenir inscrit dans une histoire collective et soumis dans le même temps à une pression forte, celle d'être l'élément pérenne de l'institution familiale. Cette distribution des cartes, tant dans l'espace intrafamilial que dans la société, nous questionne sur les modalités « de prendre sa place et de rester à sa place »d'enfant. Ce qui se joue au temps de l'adolescence, comme moment de l'émancipation et de la construction de soi, tend à se déplacer vers le temps de l'enfance, parfois dans une confusion des hiérarchies et du sens donné à l'autonomie et aux droits de l'enfant.

Fractal, Rev. Psicol., v. 27 - n. 3, p. 281-285, 2015 


\section{Références}

AUSTIN, J-L. Quand dire, c'est faire (1962). Paris: Seuil, 1991.

BOWLBY, J. Attachement et perte. Vol 1, L'attachement. Paris: PUF, 2002.

CADORET, A. Des parents comme les autres: homosexualité de parenté. Paris : Odile Jacob, 2002.

CASTORIADIS, C. L'institution imaginaire de la société (1975). Paris: Seuil, 1999.

DIASIO, N. Comment l'enfant fait-il la famille? Revue des Sciences Sociales, n. 41, 2009. "Désirs de famille, désirs d'enfant".

ELIAS, N. La société des individus. Paris: Fayard, 1989.

FRANCE. Loi $n^{\circ}$ 2002-305 du 4 mars 2002 relative à l'autorité parentale. 2002. Disponible en $\quad$ : < <ttp://www.legifrance.gouv.fr/affichTexte, do? cidTexte $=$ JORFTEXT000000776352\& $\&$ ategorieLien $=\mathrm{id}>$. Consulté le : 10 set. 2015.

GAUCHET, M. L'enfant du désir. Le Débat, n. 132, p. 98-121, 2004. Dossier "L'enfant-problème".

GIRARD, A. Situation de la famille contemporaine. Économie et Humanisme, $1^{\text {er }}$ semestre, p. 3-48, 1957.

LOURAU, R. L'analyse institutionnelle. Paris: Minuit, 1970.

MEIRIEU, P. École maternelle, école première. In : Congrès de l'AGEEM, Tarbes, 5 juil. 2008. Disponible en : <http://www meirieu.com/ARTICLES/ecole_maternelle_ecole_premiere. htm>. Consulté le : 10 set. 2015.

NEYRAND, G. Ambiguïtés de la valorisation de la coéducation à une époque de sur-responsabilisation parentale. Recherches familiales, n. 12, p. 279-287, 2015. Disponible en : <www. cairn.info/revue-recherches-familiales-2015-1-page-279.htm>. Consulté le : 10 set. 2015

REVAULT D'ALLONNES, C. et al. La démarche clinique en sciences humaines. Paris: Dunod, 1989.

SEGALEN, M. A qui appartiennent les enfants? Paris: Tallandier, 2010.

SELLENET, C. La parentalité décryptée. Paris: L'Harmattan, 2012.

WEBER, F. Manuel de l'ethnographe. Paris: PUF, 2009.

WINNICOTT, D. Jeu et réalité (1971). Paris: Gallimard, 2002.

Reçu: Septembre 2, 2015

Accepté: Octobre 5, 2015 